\title{
Spatially variable hydrologic impact and biomass production tradeoffs associated with Eucalyptus cultivation for biofuel production in Entre Rios, Argentina
}

5 Azad Heidari ${ }^{1}$, David Watkins, Jr. ${ }^{1}$, Alex Mayer ${ }^{1}$, Tamara Propato ${ }^{2}$, Santiago Verón ${ }^{2}$, Diego de Abelleyra $^{3}$

${ }^{1}$ Department of Civil and Environmental Engineering, Michigan Technological University, Houghton, 49931, USA

${ }^{2}$ INTA, Instituto Nacional de Tecnología Agropecuaria, Argentina CONICET, Consejo Nacional de Investigaciones Científicas y Técnicas, Argentina

10 FAUBA, Facultad de Agronomía de la Universidad de Buenos Aires, Buenos Aires, Argentina

${ }^{3}$ INTA, Instituto Nacional de Tecnología Agropecuaria, Argentina, Buenos Aires, Argentina

Correspondence to: David Watkins, Jr. (dwatkins@mtu.edu)

Supplemental Information.
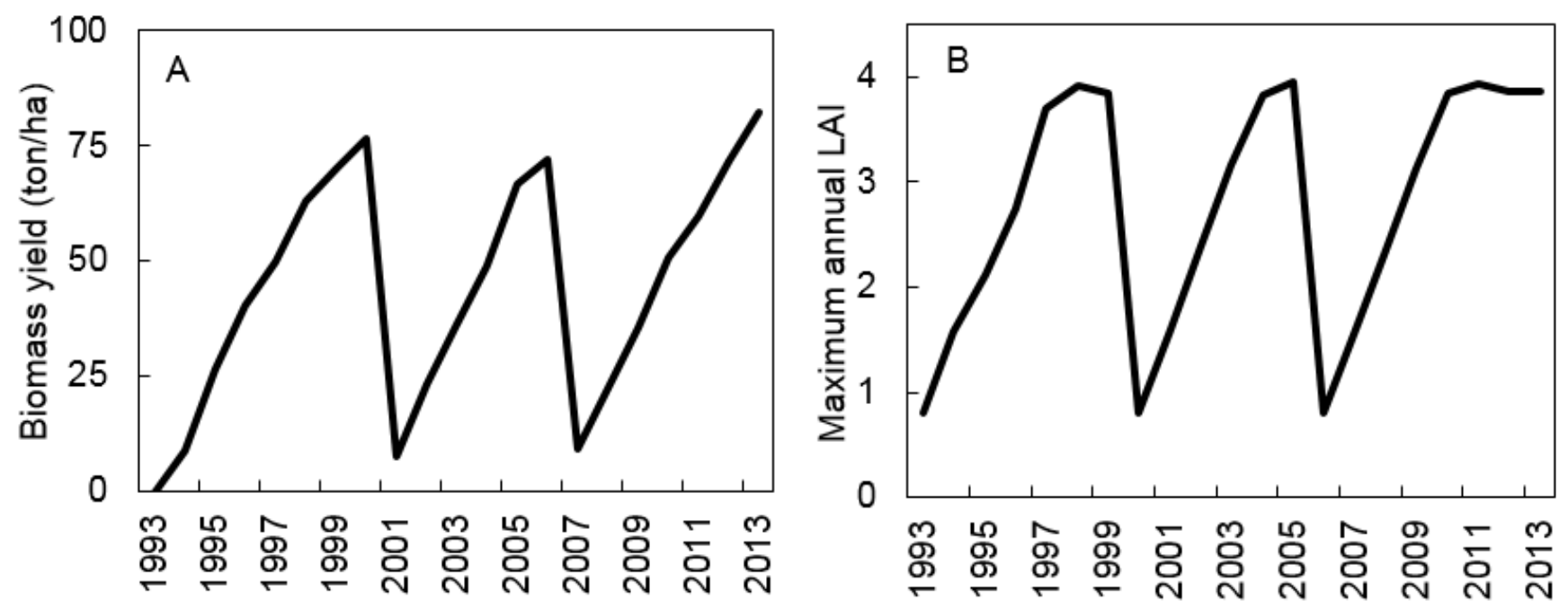

Figure S1: A) Simulated annual biomass production for the intensive scenario, and B) Simulated LAI development for the intensive scenario. 
Table S1: Land cover/land use change during the period 2002-2013.

Land cover portion of the watershed

\begin{tabular}{cccc} 
Land Cover & 2002 & 2005 & 2013 \\
\hline Orchard-Orange & $4.9 \%$ & $3.5 \%$ & $1.1 \%$ \\
Agriculture & $20.2 \%$ & $23.4 \%$ & $21.7 \%$ \\
Mixed Forest & $12.0 \%$ & $6.5 \%$ & $3.6 \%$ \\
Eucalyptus & $10.9 \%$ & $13.0 \%$ & $22.0 \%$ \\
Rangelands & $34.5 \%$ & $36.2 \%$ & $34.2 \%$ \\
Wetlands and rivers & $17.4 \%$ & $17.4 \%$ & $17.4 \%$ \\
\hline
\end{tabular}

5

Table S2: Modified parameters for hydrological model calibration and their final values.

\begin{tabular}{|c|c|c|c|c|}
\hline Parameter & Description & $\begin{array}{l}\text { Default } \\
\text { value }\end{array}$ & Final value & Range \\
\hline CN2 & SCS runoff curve number & 80 & 56.9 & $+/-30 \%$ \\
\hline $\mathrm{CH}_{-} \mathrm{K} 2$ & Effective hydraulic conductivity $(\mathrm{mm} / \mathrm{hr})$ & 0 & 16.8 & $0-25$ \\
\hline$A L P H A \_B F$ & Baseflow alpha factor (days) & 0.048 & 0.75 & $0.01-0.9$ \\
\hline$A L P H A \_B F \_D$ & Baseflow alpha factor for deep aquifer (days) & 0.01 & 0 & $0-0.1$ \\
\hline GWQmin & $\begin{array}{l}\text { Threshold depth of water in the shallow aquifer } \\
\text { required for return flow to occur }\left(\mathrm{mm} \mathrm{H}_{2} \mathrm{O}\right)\end{array}$ & 1000 & 1060.5 & $200-2000$ \\
\hline Rchrg_dp & Deep aquifer percolation fraction & 0.05 & 0.62 & $0-1$ \\
\hline CANMAX_Euca & Maximum Canopy Storage $\left(\mathrm{mm} \mathrm{H}_{2} \mathrm{O}\right)$ & 0 & 4 & $0-10$ \\
\hline CANMAX_Oran & Maximum Canopy Storage $\left(\mathrm{mm} \mathrm{H}_{2} \mathrm{O}\right)$ & 0 & 4 & $0-10$ \\
\hline
\end{tabular}


5 Table S3: Plant growth parameters in SWAT database used for eucalyptus growth simulation.

\begin{tabular}{|c|c|c|c|}
\hline Parameter & Description & $\begin{array}{l}\text { Default } \\
\text { value }\end{array}$ & $\begin{array}{l}\text { Final } \\
\text { Value }\end{array}$ \\
\hline BLAI & Maximum potential leaf area index & 2.5 & 4 \\
\hline FRGRW1 & $\begin{array}{l}\text { Fraction of the plant growing season corresponding to the } 1^{\text {st }} \text { point on the } \\
\text { optimal leaf area development curve }\end{array}$ & 0.1 & 0.05 \\
\hline LAIMX1 & $\begin{array}{l}\text { Fraction of the maximum leaf area index corresponding to the } 1^{\text {st }} \text { point on } \\
\text { the optimal leaf area development curve }\end{array}$ & 0.15 & 0.05 \\
\hline FRGWRW2 & $\begin{array}{l}\text { Fraction of the plant growing season corresponding to the } 2^{\text {nd }} \text { point on the } \\
\text { optimal leaf area development curve }\end{array}$ & 0.5 & 0.2 \\
\hline LAIMX2 & $\begin{array}{l}\text { Fraction of the maximum leaf area index corresponding to the } 2^{\text {nd }} \text { point on } \\
\text { the optimal leaf area development curve }\end{array}$ & 0.75 & 0.95 \\
\hline HVSTI & Harvest index for optimal growing condition. & 0.1 & 1 \\
\hline$D L A I$ & Fraction of growing season when leaf area begins to decline & 0.99 & 1 \\
\hline Mat_yrs & Number of years required for trees to reach maturity & 10 & 5 \\
\hline Bio_E & Radiation use efficiency & 15 & 26 \\
\hline EXT_coef & Light extinction coefficient & 0.65 & 0.4 \\
\hline Bio_Leaf & Tree biomass accumulated each year that is converted to residue & 0.3 & 0.2 \\
\hline BIO_INIT & Initial dry weight biomass (kg/ha) & 0 & 0.5 \\
\hline CURYR_MAT & Current age of trees (years) at time of planting & 0 & 1 \\
\hline
\end{tabular}

Table S4: Calibration and validation statistics for streamflow simulations.

\begin{tabular}{ccccccc}
\hline Time step & \multicolumn{2}{c}{$\begin{array}{c}\text { Calibration Period } \\
(1993-2005)\end{array}$} & \multicolumn{4}{c}{$\begin{array}{c}\text { Validation period } \\
(2005-2013)\end{array}$} \\
\cline { 2 - 7 } & \multicolumn{4}{c}{ Metric } \\
\hline \multirow{3}{*}{ Monthly } & $N S$ & Pbias & $R S q$ & $N S$ & Pbias & $R S q$ \\
& 0.58 & -7.60 & 0.58 & 0.62 & 6.77 & 0.51 \\
\hline
\end{tabular}


Table S5: Results of the optimization model to select combinations of sub-basins for maximum biomass and limited water impact.

\begin{tabular}{|c|c|c|c|c|c|c|c|c|c|c|c|c|c|}
\hline $\begin{array}{l}\text { Solution } \\
\text { Number }\end{array}$ & $\begin{array}{l}\text { Additional } \\
\text { Biomass } \\
\text { (above Base } \\
\text { case) } \\
\left(10^{6} \text { ton) }\right.\end{array}$ & $\begin{array}{l}\text { Total } \\
\text { Biomass } \\
\left(10^{6} \text { ton }\right)\end{array}$ & $\begin{array}{l}\text { Yield } \\
\text { (ton/ ha/ } \\
\text { rotation) }\end{array}$ & $\begin{array}{l}\text { Area } \\
\text { Eucalyptus } \\
\left(\mathrm{km}^{2}\right)\end{array}$ & $\begin{array}{l}\% \text { Change } \\
\text { in total flow }\end{array}$ & $\mathrm{A}$ & B & $\mathrm{C}$ & $\mathrm{D}$ & $E$ & $\mathrm{~F}$ & G & $\mathrm{H}$ \\
\hline 1 & 0.3 & 1.9 & 77.9 & 79.9 & $-1.1 \%$ & 0 & 0 & 0 & 0 & 1 & 0 & 0 & 0 \\
\hline 2 & 0.6 & 2.1 & 74.8 & 93.9 & $-2.5 \%$ & 0 & 0 & 0 & 0 & 0 & 1 & 0 & 0 \\
\hline 3 & 0.8 & 2.4 & 74.4 & 107.1 & $-2.7 \%$ & 0 & 0 & 1 & 0 & 0 & 0 & 0 & 0 \\
\hline 4 & 1.0 & 2.5 & 76.7 & 109.8 & $-3.0 \%$ & 1 & 0 & 0 & 0 & 0 & 0 & 0 & 0 \\
\hline 5 & 1.2 & 2.7 & 76.2 & 118.4 & $-3.8 \%$ & 0 & 0 & 1 & 0 & 1 & 0 & 0 & 0 \\
\hline 6 & 1.3 & 2.8 & 78.2 & 121.1 & $-4.2 \%$ & 1 & 0 & 0 & 0 & 1 & 0 & 0 & 0 \\
\hline 7 & 1.6 & 3.1 & 72.8 & 144.0 & $-5.3 \%$ & 0 & 1 & 0 & 0 & 1 & 0 & 0 & 0 \\
\hline 8 & 1.8 & 3.4 & 75.7 & 148.3 & $-5.7 \%$ & 1 & 0 & 1 & 0 & 0 & 0 & 0 & 0 \\
\hline 9 & 1.8 & 3.4 & 75.9 & 149.0 & $-6.1 \%$ & 0 & 0 & 0 & 1 & 0 & 0 & 0 & 0 \\
\hline 10 & 2.1 & 3.7 & 76.9 & 159.6 & $-6.9 \%$ & 1 & 0 & 1 & 0 & 1 & 0 & 0 & 0 \\
\hline 11 & 2.3 & 3.8 & 72.9 & 173.9 & $-7.2 \%$ & 1 & 1 & 0 & 0 & 0 & 0 & 0 & 0 \\
\hline 12 & 2.7 & 4.2 & 75.3 & 187.5 & $-8.8 \%$ & 0 & 0 & 1 & 1 & 0 & 0 & 0 & 0 \\
\hline 13 & 3.1 & 4.6 & 72.9 & 212.4 & $-9.9 \%$ & 1 & 1 & 1 & 0 & 0 & 0 & 0 & 0 \\
\hline 14 & 3.1 & 4.7 & 77.5 & 201.5 & $-10.3 \%$ & 1 & 0 & 0 & 1 & 1 & 0 & 0 & 0 \\
\hline 15 & 3.7 & 5.2 & 75.9 & 228.7 & $-11.8 \%$ & 1 & 0 & 1 & 1 & 0 & 0 & 0 & 0 \\
\hline 16 & 4.0 & 5.5 & 76.8 & 240.0 & $-13.0 \%$ & 1 & 0 & 1 & 1 & 1 & 0 & 0 & 0 \\
\hline 17 & 4.1 & 5.6 & 74.0 & 254.3 & $-13.3 \%$ & 1 & 1 & 0 & 1 & 0 & 0 & 0 & 0 \\
\hline 18 & 4.4 & 6.0 & 74.8 & 265.6 & $-14.4 \%$ & 1 & 1 & 0 & 1 & 1 & 0 & 0 & 0 \\
\hline 19 & 4.9 & 6.5 & 73.8 & 292.8 & $-16.0 \%$ & 1 & 1 & 1 & 1 & 0 & 0 & 0 & 0 \\
\hline 20 & 5.3 & 6.8 & 74.6 & 304.1 & $-17.1 \%$ & 1 & 1 & 1 & 1 & 1 & 0 & 0 & 0 \\
\hline 21 & 5.5 & 7.0 & 73.8 & 318.1 & $-18.5 \%$ & 1 & 1 & 1 & 1 & 0 & 1 & 0 & 0 \\
\hline 22 & 5.8 & 7.4 & 74.5 & 329.4 & $-19.6 \%$ & 1 & 1 & 1 & 1 & 1 & 1 & 0 & 0 \\
\hline 23 & 6.0 & 7.5 & 75.9 & 331.4 & $-21.1 \%$ & 1 & 1 & 1 & 1 & 1 & 0 & 1 & 0 \\
\hline 24 & 6.2 & 7.8 & 75.2 & 345.4 & $-22.4 \%$ & 1 & 1 & 1 & 1 & 0 & 1 & 1 & 0 \\
\hline 25 & 6.6 & 8.1 & 75.7 & 356.7 & $-23.5 \%$ & 1 & 1 & 1 & 1 & 1 & 1 & 1 & 0 \\
\hline 26 & 6.7 & 8.3 & 76.1 & 363.7 & $-24.8 \%$ & 1 & 1 & 1 & 1 & 1 & 1 & 0 & 1 \\
\hline 27 & 6.9 & 8.5 & 77.3 & 365.7 & $-26.3 \%$ & 1 & 1 & 1 & 1 & 1 & 0 & 1 & 1 \\
\hline
\end{tabular}




\begin{tabular}{|c|c|c|c|c|c|c|c|c|c|c|c|c|c|}
\hline 28 & 7.2 & 8.7 & 76.6 & 379.7 & $-27.6 \%$ & 1 & 1 & 1 & 1 & 0 & 1 & 1 & 1 \\
\hline 29 & 7.5 & 9.0 & 77.1 & 391.0 & $-28.8 \%$ & 1 & 1 & 1 & 1 & 1 & 1 & 1 & 1 \\
\hline Times & & & & & & 22 & 16 & 19 & 18 & 15 & 8 & 6 & 4 \\
\hline Selecte & & & & & & & & & & & & & \\
\hline
\end{tabular}

